\title{
Erratum: Hyperon stars in a modified quark meson coupling model [Phys. Rev. C 94, 035805 (2016)]
}

\author{
R. N. Mishra, H. S. Sahoo, P. K. Panda, N. Barik, and T. Frederico
}

(Received 1 May 2018; revised manuscript received 29 May 2018; published 9 July 2018)

DOI: 10.1103/PhysRevC.98.019903

In the original paper, we had developed the equation of state (EOS) of nuclear matter with the inclusion of hyperons in a modified quark meson coupling model. In one of the conclusions we computed the radius of the neutron star corresponding to the maximum mass and the canonical mass of $1.44 M_{\odot}$. We observed an error in variable substitution in the computation of the equation of state for the baryon octet. The correct computation alters the results that we have displayed in Tables V, VI, and columns 7-9 of Table IV of the published paper. Accordingly the figures, Figs. 2, 4, 5, and 8 of the original paper get modified. All the above tables and figures referring to Sec. V: "Results and Discussions" of the published paper are corrected and are presented in this Erratum. In Table IV of the original paper, the value of nuclear matter incompressibility $K$ for the quark masses $m_{q}=80$ and $m_{q}=150 \mathrm{MeV}$ was determined to be 246 and $292 \mathrm{MeV}$, respectively. After the correction we observe a change in the values of $K$, saturation field values of the $\sigma$ meson $\left(\sigma_{0}\right)$, and the ratio $\frac{M_{N}^{*}}{M_{N}}$. The corresponding columns in Table IV are revised, and the corrected values are shown. All other values of Table IV in the original paper remain unchanged. The revised hyperon couplings to the $\omega$ meson $x_{\omega B}$ are given in Table $\mathrm{V}$.

The corrected figure for the effective mass of the baryons as a function of baryon density is given in Fig. 2, and the corrected figure for the EOS at various cascade potentials and at different values of the coupling parameter $\Lambda_{v}$ is shown in Fig. 4 .

The corrected $x_{\omega B}$ impacts the density of formation of the hyperons. In the corrected figure shown in Fig. 5, we observe the appearance of $\Lambda$ baryon first followed by $\Xi^{-}$. Furthermore, it is observed in the revised Fig. 7 that the strangeness fraction increases at lower densities as compared to the earlier published version, indicating a higher strangeness content and a softer EOS. Figure 8 shows the corrected mass-radius relations. Setting the least attractive cascade potential at $U_{\Xi}=0 \mathrm{MeV}$, the maximum masses of a neutron star for quark masses $m_{q}=80$ and $m_{q}=150 \mathrm{MeV}$ after correction are obtained as $1.66 M_{\odot}$ and $1.86 M_{\odot}$, respectively. Increasing the quark mass to $m_{q}=200 \mathrm{MeV}$, we obtain a maximum mass of $1.96 M_{\odot}$ whereas the quark mass of $m_{q}=250 \mathrm{MeV}$ gives $2.16 M_{\odot}$. The results for $m_{q}=200$ and $m_{q}=250 \mathrm{MeV}$ are given in the revised Table VI.

The corrected radius corresponding to the maximum mass varies within $11.0-12.9 \mathrm{~km}$, and the radius corresponding to the canonical mass $1.44 M_{\odot}$ varies within a range of $11.9-13.9 \mathrm{~km}$ for different sets of the parameters which include that of quark masses $m_{q}=80, m_{q}=150, m_{q}=200$, and $m_{q}=250 \mathrm{MeV}$. The revised results are given here in Table VI. It is to be noted that, with the corrected computation, the $2 M_{\odot}$ constraint with the chosen quark mass $m_{q}=150 \mathrm{MeV}$ is not coming up. We are able to achieve the mass constraint set by PSRJ1614-2230 and PSR J0348+0432 by increasing the quark mass to $m_{q}=200 \mathrm{MeV}$ and more. Unlike the conclusion in the original paper, it is found that with the corrected results the radius corresponding to the canonical mass of $1.44 M_{\odot}$ is within the predicted values.

TABLE IV. Parameters for nuclear matter. They are determined from the binding energy per nucleon $E_{B . E}=B_{0} \equiv \mathcal{E} / \rho_{B}-M_{N}=$ $-15.7 \mathrm{MeV}$ and pressure $P=0$ at saturation density $\rho_{B}=\rho_{0}=0.15 \mathrm{fm}^{-3}$. Also shown are the values of the nuclear matter incompressibility $K$ and the slope of the symmetry energy $L$ for the quark masses $m_{q}=80$ and $m_{q}=150 \mathrm{MeV}$.

\begin{tabular}{|c|c|c|c|c|c|c|c|c|c|c|c|}
\hline \multirow{2}{*}{$\begin{array}{l}m_{q} \\
(\mathrm{MeV})\end{array}$} & \multirow[t]{2}{*}{$g_{\sigma}^{q}$} & \multirow[t]{2}{*}{$g_{\omega}$} & \multicolumn{3}{|c|}{$g_{\rho}$} & \multirow{2}{*}{$\begin{array}{c}\sigma_{0} \\
(\mathrm{MeV})\end{array}$} & \multirow[t]{2}{*}{$\frac{M_{N}^{*}}{M_{N}}$} & \multirow{2}{*}{$\begin{array}{c}K \\
(\mathrm{MeV})\end{array}$} & \multicolumn{3}{|c|}{$L(\mathrm{MeV})$} \\
\hline & & & $\Lambda_{v}=0$ & $\Lambda_{v}=0.05$ & $\Lambda_{v}=0.1$ & & & & $\Lambda_{v}=0$ & $\Lambda_{v}=0.05$ & $\Lambda_{v}=0.1$ \\
\hline 150 & 4.39952 & 6.74299 & 8.79976 & 9.2522 & 9.7825 & 20.92 & 0.85 & 237 & 86.39 & 92.45 & 99.95 \\
\hline
\end{tabular}

TABLE V. The corrected results for $x_{\omega B}$ determined by fixing the potentials for the hyperons.

\begin{tabular}{|c|c|c|c|c|c|}
\hline $\begin{array}{l}m_{q} \\
(\mathrm{MeV})\end{array}$ & $\frac{x_{\omega \Lambda}}{U_{\Lambda}=-28 \mathrm{MeV}}$ & $\frac{x_{\omega \Sigma}}{U_{\Sigma}=30 \mathrm{MeV}}$ & $U_{\Xi}=-18 \mathrm{MeV}$ & $U_{\Xi}=-10 \mathrm{MeV}$ & $U_{\Xi}=0 \mathrm{MeV}$ \\
\hline 150 & 0.80443 & 1.54049 & 0.40363 & 0.49722 & 0.61423 \\
\hline
\end{tabular}


TABLE VI. Stellar properties obtained at different values of the parameter $\Lambda_{v}$ and the $\Xi$-meson coupling for quark masses $m_{q}=80, m_{q}=$ $150, m_{q}=200$, and $m_{q}=250 \mathrm{MeV}$.

\begin{tabular}{|c|c|c|c|c|c|c|}
\hline \multirow[t]{4}{*}{80} & -18 & 0 & 5.11 & 1.58 & 12.1 & 13.0 \\
\hline & -18 & 0.1 & 6.32 & 1.44 & 11.2 & 11.9 \\
\hline & -10 & 0 & 5.59 & 1.63 & 11.8 & 13.0 \\
\hline & 0 & 0 & 6.21 & 1.66 & 11.2 & 13.0 \\
\hline \multirow{6}{*}{150} & -18 & 0.05 & 5.71 & 1.67 & 11.6 & 12.8 \\
\hline & -18 & 0.1 & 6.27 & 1.66 & 11.2 & 12.3 \\
\hline & -10 & 0 & 4.90 & 1.82 & 12.4 & 13.5 \\
\hline & -10 & 0.05 & 5.99 & 1.72 & 11.5 & 12.8 \\
\hline & -10 & 0.1 & 6.49 & 1.71 & 11.0 & 12.3 \\
\hline & 0 & 0 & 5.27 & 1.86 & 12.2 & 13.5 \\
\hline 200 & 0 & 0 & 4.91 & 1.96 & 12.5 & 13.7 \\
\hline \multirow{3}{*}{250} & -18 & 0 & 4.54 & 2.12 & 12.9 & 13.9 \\
\hline & -10 & 0 & 4.71 & 2.14 & 12.8 & 13.9 \\
\hline & 0 & 0 & 4.48 & 2.16 & 12.7 & 13.9 \\
\hline
\end{tabular}

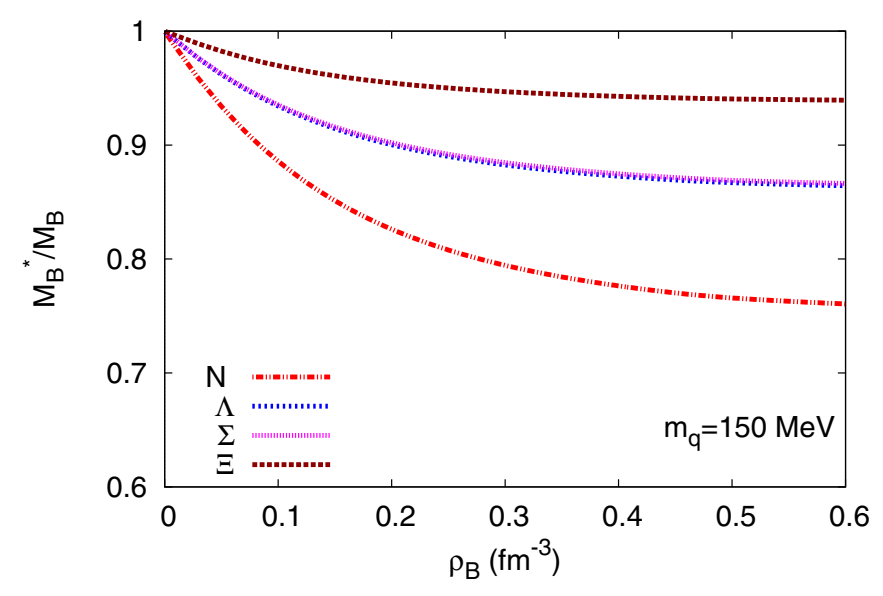

FIG. 2. Effective mass of the baryon at quark mass $m_{q}=150 \mathrm{MeV}$.

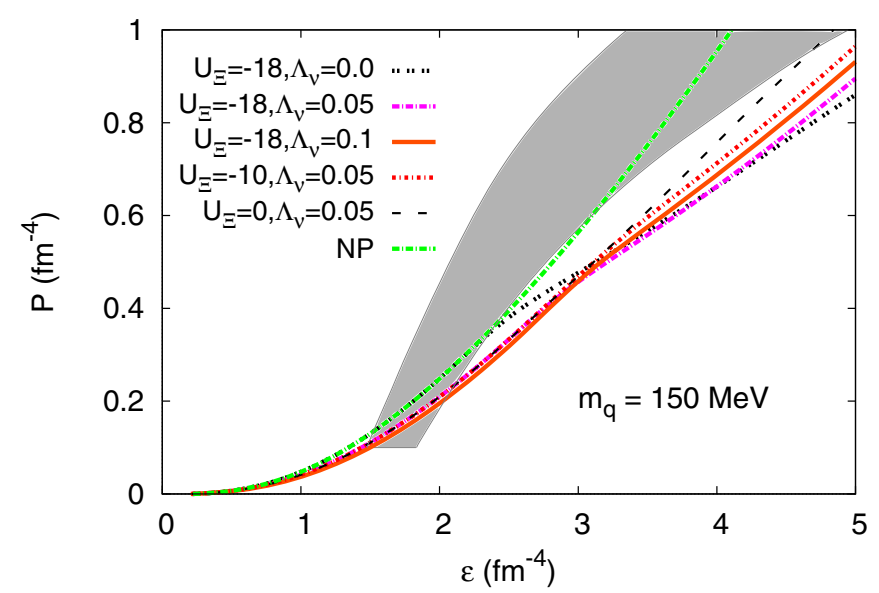

FIG. 4. The EOS at various cascade potentials and different values of $\Lambda_{v}$ for quark mass $m_{q}=150 \mathrm{MeV}$. The shaded region shows the empirical EOS obtained by Steiner et al. from a heterogeneous set of seven neutron stars. 

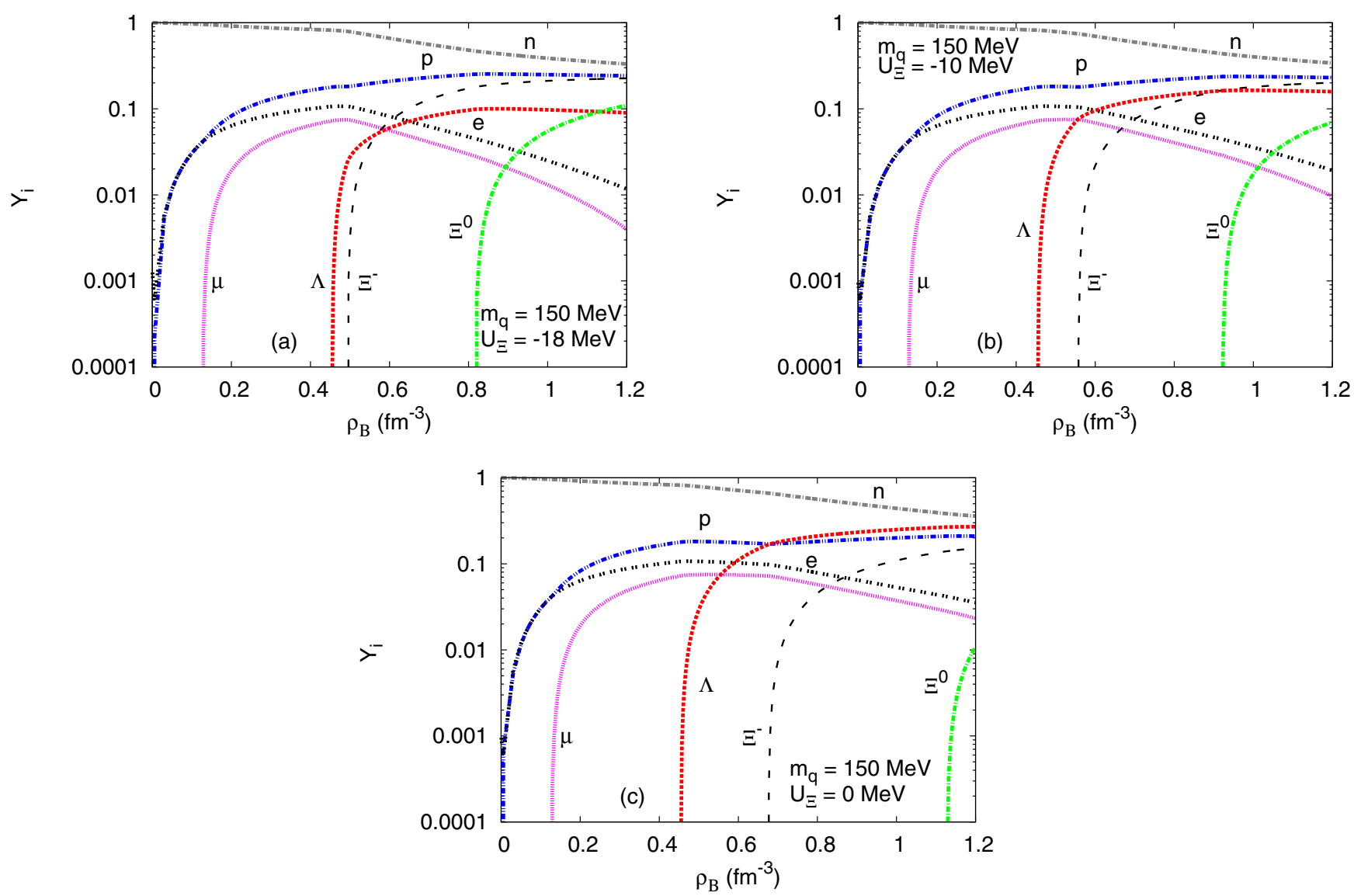

FIG. 5. Particle fraction at different cascade potentials of (a) $U_{\Xi}=-18 \mathrm{MeV}$, (b) $U_{\Xi}=-10 \mathrm{MeV}$, and (c) $U_{\Xi}=0 \mathrm{MeV}$ for the quark mass $m_{q}=150 \mathrm{MeV}$.

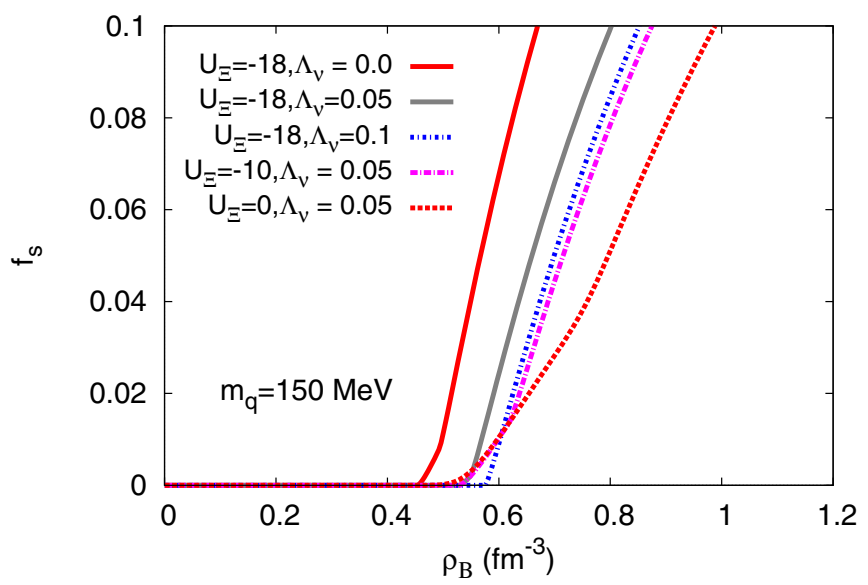

FIG. 7. Strangeness fraction as a function of density for various cascade potentials. 

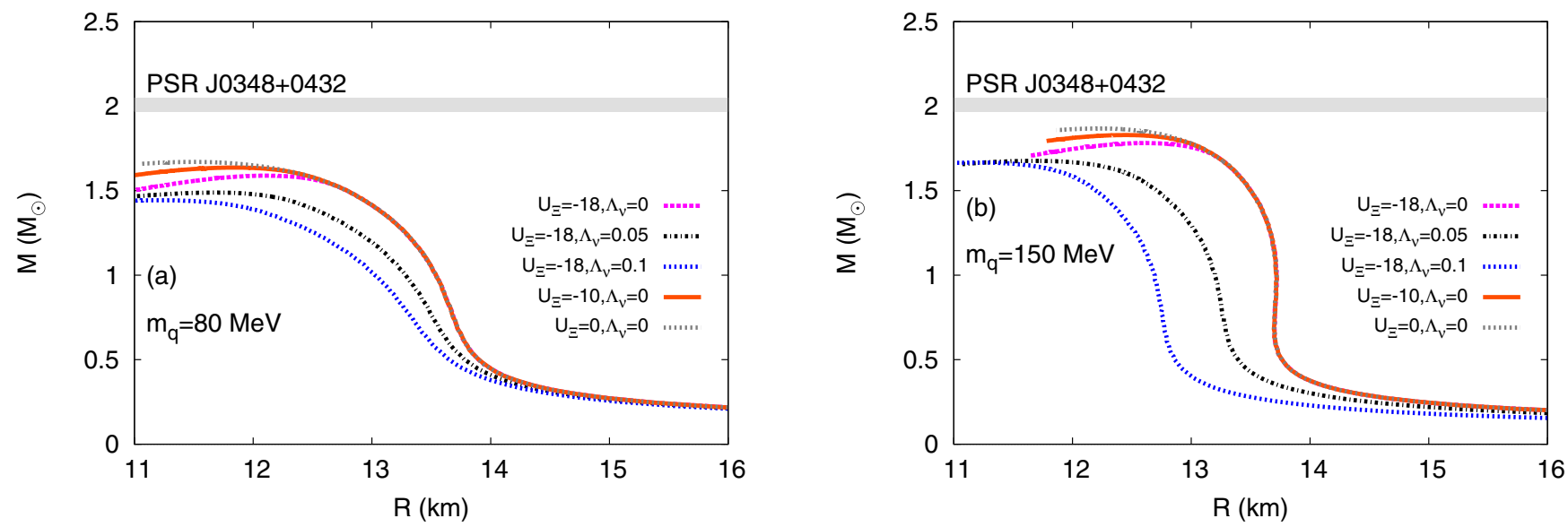

FIG. 8. Corrected star mass as a function of radius for various values of the coupling parameter and cascade potential at quark masses (a) $m_{q}=80 \mathrm{MeV}$ and (b) $m_{q}=150 \mathrm{MeV}$. Also shown is the mass observed for the pulsar PSR J0348+0432. 